\title{
A damage identification procedure based on Hilbert transform: Experimental validation
}

\author{
F. Lo Iacono ${ }^{1}$, G. Navarra ${ }^{2}$ and A. Pirrotta ${ }^{1, *, \dagger}$ \\ ${ }^{1}$ Dipartimento di Ingegneria Strutturale Aerospaziale e Geotecnica (DISAG), Università degli Studi di Palermo, \\ viale delle Scienze I-90128 Palermo, Italy \\ ${ }^{2}$ Libera Università di Enna 'Kore', Cittadella Universitaria I-94100 Enna, Italy
}

\begin{abstract}
SUMMARY
This paper aims at validating the feasibility of an identification procedure, based on the use of the Hilbert transform, by means of experimental tests for shear-type multi-degree-of-freedom systems. Particularly, a three-degree-of-freedom frame will be studied either numerically or experimentally by means of a laboratory scale model built at the laboratory of the Structural, Aerospace and Geotechnical Engineering Department (DISAG) of University of Palermo. Several damage scenarios have been considered to prove the effectiveness of the procedure. Moreover, the experimental tests have been conducted by considering two different input loads: pulse forces, simulated by means of an instrumental hammer, and wide band noise base inputs, by a shake table.

In the first section the damage identification procedure, proposed in recent works, is presented. The procedure is based on the minimization of an objective function mathematically based on the properties of the analytical signal and the Hilbert transform. Second section reports the experimental model geometrical data and the data acquisition set-up as built in the DISAG laboratory. In Section 3, the results of the experimental campaigns are presented and discussed having considered three damage scenarios. The validated procedure has been proved to be able to not only detect damage even at early stage but it also needs processing of only few samples of the structural response. Copyright (C) 2010 John Wiley \& Sons, Ltd.
\end{abstract}

Received 13 January 2010; Revised 23 September 2010; Accepted 17 October 2010

KEY WORDS: Hilbert transform; analytical signal; incipient damage

\section{INTRODUCTION}

In the past forty years many researchers have focused on the problem of damage identification aiming at providing a reliable damage identification procedure or a structural health monitoring program. Basically, methods reported in literature can be classified into three categories: experimental-data-based methods, model-based methods and model-free methods based. Regarding experimental-data-based methods, the analysis of a recorded signal leads one to identify the presence of probable damage, especially from a comparison with signals of the healthy structure. Structural checking may be based on natural frequencies [1-3], damping ratios [4], modal data [5,6], curvature measures [7-9], frequency response functions (FRFs) [10-13] and strain energy $[14,15]$. The advantage of the experimental-data-based methods is that a mathematical model such as a refined finite element model (FEM) of the structure is not

\footnotetext{
*Correspondence to: A. Pirrotta, Dipartimento di Ingegneria Strutturale Aerospaziale e Geotecnica (DISAG), Università degli Studi di Palermo, viale delle Scienze I-90128 Palermo, Italy.

†E-mail: antonina.pirrotta@unipa.it
} 
required. In model-based methods, it is assumed that a FEM of the structure is available before damage has occurred and that the FEM and the experimental modal analysis model can be matched under certain criteria for the undamaged structure; among model-based methods, we can consider optimal matrix modifications [16-18] and sensitivity-based updates [19]. Modelfree detection methods are based on artificial neural networks [20,21].

In [22] a damage identification procedure, proper for highlighting very low level of damage, has been performed. When dealing with a very low level of damage, for instance, a little variation in structural stiffness, this phenomenon cannot be detected either in time domain or in frequency domain, in fact in [23], it was stressed that the healthy impulse response function (IRF) and the healthy frequency response function (FRF) totally overlaps the IRF or the FRF of the damaged one; this indicates that both IRF and FRF are not 'good features' for low damage identification procedure. Then an identification procedure very useful to detect low variation of structural stiffness was developed. In this regard, this procedure is based on applying Hilbert transform to obtain the analytical representation of the system response to a given input (pulse force load and wide band noise). The characteristics of the response analytical signal (frequency, phase and amplitude) have been proved to be very sensitive even to very small variation strictly connected to the structural stiffness. According to the above considerations, this indicates that the characteristics of analytical signal are 'good features' for low damage identification procedure. Moreover in [23] the same identification procedure was demonstrated to be valid also in the identification of entity and localization of multiple simultaneous damages.

This paper aims at validating the feasibility of the aforementioned procedure through experimental tests conducted in the laboratory of the Structural, Aerospace and Geotechnical Engineering Department (DISAG) of the University of Palermo.

First the damage identification procedure developed in [22] has been reported, considering a structure excited by an pulse force. Second, once the experimental setup is described, the results for various cases of damage scenarios are reported. Finally, it has been shown that the proposed procedure not only is able to detect the damage pattern even at an early stage but it also needs processing of only few samples of the structural response.

\section{DAMAGE IDENTIFICATION PROCEDURE}

The results of the damage identification procedure, developed in [22], are able to detect and localize [23] a damage even at an early stage.

The main idea was to detect the damage by evaluating a properly defined objective function, whose general expression may be given in the form:

$$
J_{\eta}(\boldsymbol{\alpha})=\frac{\int_{T_{\text {ini }}}^{T_{\text {fin }}}\left[\eta^{\text {th }}(\boldsymbol{\alpha}, t)-\eta^{\mathrm{ex}}(t)\right]^{2} \mathrm{~d} t}{\int_{T_{\text {ini }}}^{T_{f i n}}\left[\eta^{\mathrm{ex}}(t)\right]^{2} \mathrm{~d} t}
$$

where $\left[T_{\mathrm{fin}}-T_{\mathrm{ini}}\right]$ is the observation window, $\boldsymbol{\alpha}$ is a vector casting the various damage parameters $\alpha_{i}, \eta(t)$ denotes a generic response feature, while the apexes th and $e x$ stand for theoretical and experimentally measured, respectively. Moreover, $\eta^{\operatorname{ex}}(t)$ stands for the experimental measurement of these features in the damaged system, while $\eta^{\text {th }}(\boldsymbol{\alpha}, t)$ has to be intended as the numerical evaluation of the same features for different values of damage parameters $\alpha_{i}$, once a numerical model of the system has been considered.

In other words, the objective function $J_{\eta}(\boldsymbol{\alpha})$ exhibits a minimum in correspondence of a damage pattern $\boldsymbol{\alpha}$ which identifies a system, within the chosen class of mechanical systems, such that the difference among the simulated response characteristics $\eta^{\text {th }}(\boldsymbol{\alpha}, t)$ and the experimental response characteristic $\eta^{\mathrm{ex}}(t)$ is minimum.

In literature several different choices for the response feature may be found, such as kinematic features (displacement, velocity or acceleration) and derived ones (FRF, modal shape, modal curvature etc.) as well. But, in light of previous results [22-25], it seems that the best choice for $\eta(t)$ is to use some characteristics of the analytical signal, having higher sensitivity to slight variation in system parameters. 
It is worth to note that the analytical signal of $x(t)$ is a complex valued time function defined as

$$
z(t)=x(t)+i \hat{x}(t)
$$

whose real part is the signal itself and the imaginary part $\hat{x}(t)$ is the Hilbert transform (HT) of the signal:

$$
\hat{x}(t)=\frac{1}{\pi} \int_{-\infty}^{+\infty} \frac{x(\tau)}{t-\tau} \mathrm{d} \tau
$$

the latter integral implies a Cauchy principal value. Expressing the analytical signal in polar form

$$
z(t)=A_{z}(t) \exp \left[i \theta_{z}(t)\right]
$$

the amplitude $A_{z}(t)=\sqrt{x(t)^{2}+\hat{x}(t)^{2}}$ and the phase $\theta_{z}(t)=\arctan [\hat{x}(t) / x(t)]$ can be defined. It is also worth to remind that the time derivative of the phase angle is referred to as the instantaneous frequency $\omega_{z}(t)=\dot{\theta}_{z}(t)$.

This damage identification procedure works very well in the case of single degree of freedom (SDOF), and the objective function derived following Equation (1) has a sharp minimum in correspondence of the actual damage parameters [22]; however, the identification procedure to signals, which are responses of multi-degree-of-freedom (MDOF) systems, was not satisfactory, because the signals do not have a well-behaved Hilbert transform. In fact a mono-component signal, like the SDOF response, has a well-behaved Hilbert transform and not the total response of the MDOF system, which is a multi-component signal.

In a previous work [24], to overcome the latter problem, the empirical mode decomposition (EMD) was necessary to restore the sharpness of the procedure adopted in SDOF. The EMD method, proposed by Huang [26], is widely used for signal detection [27], damage identification [28], sensitivity analysis and so on and it was demonstrated to be capable of identifying modal parameters as well as mode shapes and mass, stiffness and damping matrices of linear structures more accurately than the method based on the wavelet transforms [29-31].

Indeed, the EMD is a black-box software, based on empirical observations, hence in recent papers [22-25] a procedure has been developed for getting rid from the use of the EMD, validity of results being equal.

To do that the response signal is separated in several components, labelled filtered modal responses (FMR), by means of a proper band-pass filter around each modal frequency. In such a way we are in the same condition either as the SDOF case or using EMD for MDOF systems: a signal that has a well-behaved Hilbert Transform.

To show the feasibility of this procedure, here we have considered both numerical and experimental response of a 3-DOF shear-type model subjected to a force time history $\mathbf{F}(t)$; the equation of motion of such a system may be expressed as

$$
\mathbf{M} \ddot{\mathbf{X}}(t)+\mathbf{C} \dot{\mathbf{X}}(t)+\mathbf{K X}(t)=\mathbf{F}(t)
$$

where $\mathbf{X}(t)$ is the displacement vector, $\mathbf{C}$ is the damping matrix and $\mathbf{M}$ and $\mathbf{K}$ are the lumped mass and stiffness matrices, respectively:

$$
\mathbf{M}=\left[\begin{array}{ccc}
m_{1} & 0 & 0 \\
0 & m_{2} & 0 \\
0 & 0 & m_{3}
\end{array}\right], \quad \mathbf{K}=\left[\begin{array}{ccc}
k_{1}+k_{2} & -k_{2} & 0 \\
-k_{2} & k_{2}+k_{3} & -k_{3} \\
0 & -k_{3} & k_{3}
\end{array}\right]
$$

Furthermore, supposing the system classically damped, the following expression holds true

$$
\boldsymbol{\Phi}^{\mathrm{T}} \mathbf{C} \boldsymbol{\Phi}=\boldsymbol{\Lambda}=\left[\begin{array}{ccc}
2 \zeta_{1} \omega_{1} & 0 & 0 \\
0 & 2 \zeta_{2} \omega_{2} & 0 \\
0 & 0 & 2 \zeta_{3} \omega_{3}
\end{array}\right]
$$

where $\boldsymbol{\Phi}$ is the modal matrix, $\omega_{i}$ and $\zeta_{i}$ are the $i$ th natural frequency and modal damping ratio, respectively. The system reported in Equation (5) will be referred to as healthy structure. 
Then, let us suppose that the structure suffers a damage that can be modelled as a slight reduction in stiffness that may occur in any floor. Thus, in general, we can define a damaged stiffness matrix $\mathbf{K}_{\mathrm{D}}$ depending only on three damage parameters $\alpha_{i}, 0 \leqslant \alpha_{i} \leqslant 1(i=1,2,3)$ :

$$
\mathbf{K}_{\mathrm{D}}=\left[\begin{array}{ccc}
k_{1}\left(1-\alpha_{1}\right)+k_{2}\left(1-\alpha_{2}\right) & -k_{2}\left(1-\alpha_{2}\right) & 0 \\
-k_{2}\left(1-\alpha_{2}\right) & k_{2}\left(1-\alpha_{2}\right)+k_{3}\left(1-\alpha_{3}\right) & -k_{3}\left(1-\alpha_{3}\right) \\
0 & -k_{3}\left(1-\alpha_{3}\right) & k_{3}\left(1-\alpha_{3}\right)
\end{array}\right]
$$

Moreover, a damage is modelled in such a way that only the stiffness matrix changes, while the damping matrix of healthy and damaged structures are assumed to be equal.

The aim of the procedure is to detect the damage pattern in terms of $\alpha_{i}$ by processing few samples of acceleration time history recorded at a single point of a structure.

In particular, the healthy structure considered for both numerical and experimental data is a 3-DOF shear-type building (Figure 1), whose dynamical properties are reported in the following Table I. This structure has been excited either by an hammer pulse, or by a broadband noise with uniform power amplitude from 5 to $80 \mathrm{~Hz}$.

Moreover, each floor acceleration response $\ddot{x}_{p}(t)$ is a multi-frequency signal, and then it is necessary to extract the FMR, $\ddot{x}_{p j}(t)$, for each mode $j$, using a cascade of low pass filters, around each modal frequency.

Once all the $j$ th FMR have been evaluated and their analytical signals have been computed, the functional $J_{\eta}(\alpha)$ in Equation (1) is then calculated for each filtered modal response

$$
J_{\eta, j}(\boldsymbol{\alpha})=\frac{\int_{T_{\mathrm{ini}}}^{T_{\text {fin }}}\left[\eta_{j}^{\mathrm{th}}(\boldsymbol{\alpha}, t)-\eta_{j}^{\mathrm{ex}}(t)\right]^{2} \mathrm{~d} t}{\int_{T_{\mathrm{ini}}}^{T_{\text {fin }}}\left[\eta_{j}^{\mathrm{ex}}(t)\right]^{2} \mathrm{~d} t}
$$

And, eventually, the objective function can be obtained as

$$
J_{\eta}(\boldsymbol{\alpha})=\sum_{j=1, n} w_{j} J_{\eta, j}(\boldsymbol{\alpha})
$$

where $w_{j}$ is a suitable weight function.

In next sections the experimental set-up will be shown and the experimental results will be reported and discussed.

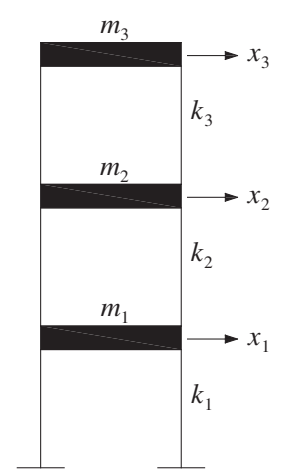

Figure 1. Simplified 3-DOF system.

Table I. Dynamical properties of the system depicted in Figure 1.

\begin{tabular}{lccccc}
\hline Floor & Mass $(\mathrm{kg})$ & Stiffness $(\mathrm{N} / \mathrm{m})$ & Mode & Frequency $(\mathrm{Hz})$ & Damping ratio $(\%)$ \\
\hline $1^{\circ}$ & 0.747 & 19249.1 & $1^{\circ}$ & 12.32 & 0.87 \\
$2^{\circ}$ & 0.755 & 25967.1 & $2^{\circ}$ & 36.04 & 0.19 \\
$3^{\circ}$ & 0.689 & 26768.0 & $3^{\circ}$ & 53.25 & 0.21 \\
\hline
\end{tabular}




\section{EXPERIMENTAL SET-UP AND DATA ACQUISITION}

The experimental model, built-up in the DISAG laboratory of the University of Palermo and depicted in Figure 2, consists of a small-scaled plane three-storey shear-type building, having the same characteristics reported in Table I. Each storey is composed of a rigid girder made of Anticorodal (an aluminium alloy), with a span of $L=350 \mathrm{~mm}$. For the healthy system, the column of all stories, represented by leaf springs with rectangular cross-section $(3 \times 15 \mathrm{~mm})$, is made of hard steel. The height of each storey is $h_{1}=200 \mathrm{~mm}$ and $h_{2}=h_{3}=180 \mathrm{~mm}$, respectively. The masses, lumped at the floors, are $m_{1}=0.747 \mathrm{~kg}, m_{2}=0.755 \mathrm{~kg}$ and $m_{3}=0.689 \mathrm{~kg}$, respectively.

The acceleration responses at each storey have been acquired using Bruel \& Kjær Type 4507-002B piezoelectric accelerometers and a PCB model 481A amplifier. Voltage signals have been generated by means of a National Instruments NI-PCI-MIO-16XE-10 and have been digitalized and acquired by means of a National Instruments NI-PCI-4472 Acquisition Board and then processed using a self-developed signal processing software in Labview and Matlab environment.

In the case of free vibration test, the excitation was supplied using a Bruel \& Kjær - Type 8202 Impact Hammer and the signal acquired from the force transducer was used as input signal. In the case of broadband noise tests, the model was fixed on a APS Dynamic-Model 133 shake table and an accelerometer on the table itself supplied the input signal.

In Figure 2(b) a picture of the experimental model setup equipped with the accelerometers and arranged to perform a broadband noise test is reported, while in Figure 3 a detail of the accelerometer mounting and of the column connection system is depicted.

In Figures 4 and 5 a schematic view of the acquisition system for free vibration test and broadband noise test are reported, respectively.

All signals have been acquired using a sampling rate equal to $1000 \mathrm{~Hz}$ and a total samples of 20000 in order to have $20 \mathrm{~s}$ acceleration time histories.

The dynamical parameters of the system have been identified by means of classical system identification procedures based on the frequency response. In particular, the experimental transfer functions of both healthy and damaged systems have been calculated for both hammer tests and broadband noise tests and then used to perform the linear system identification.

Modal frequencies, damping ratios and mode shapes matrices have been computed using several identification procedures such as Rational Polynomial Interpolation (RPI) [32] and Ibrahim Time Domain (ITD) method [33].

(a)

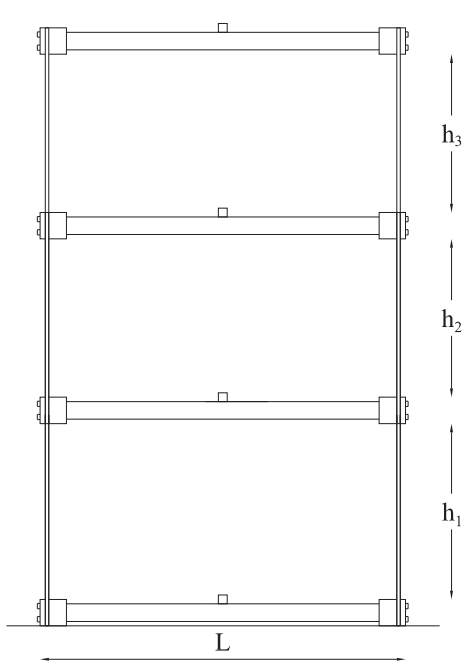

(b)

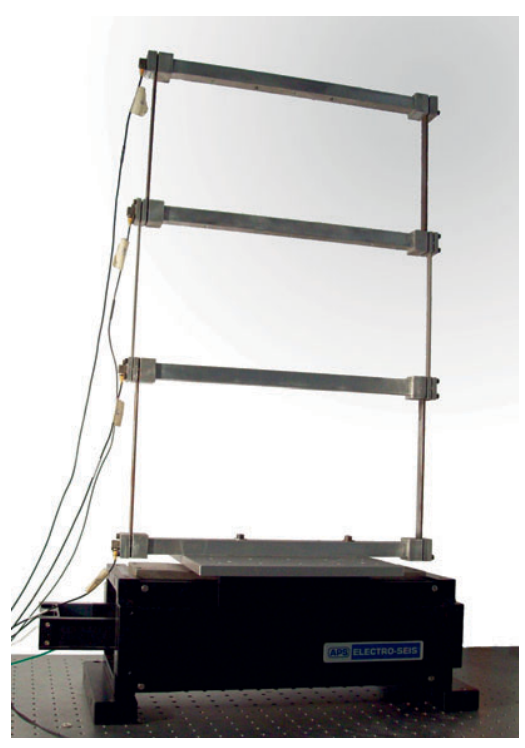

Figure 2. (a) Schematic view of the three-storey shear-type model and (b) picture of the experimental setup for broadband noise tests. 


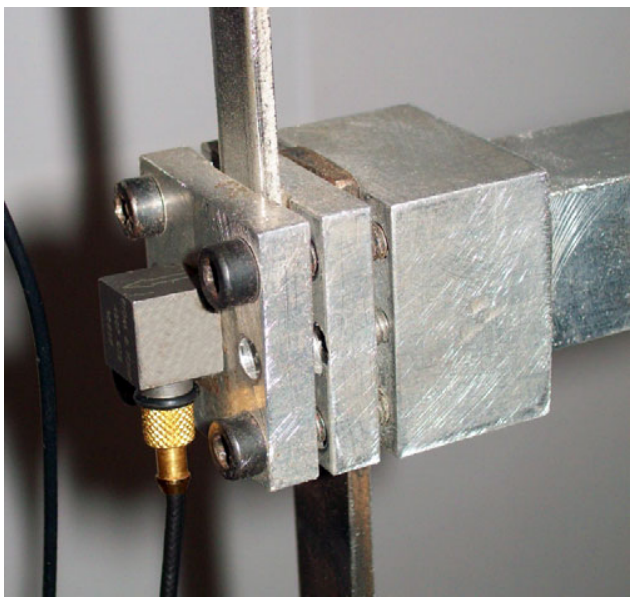

Figure 3. Detail of the accelerometer mounting and column connection.

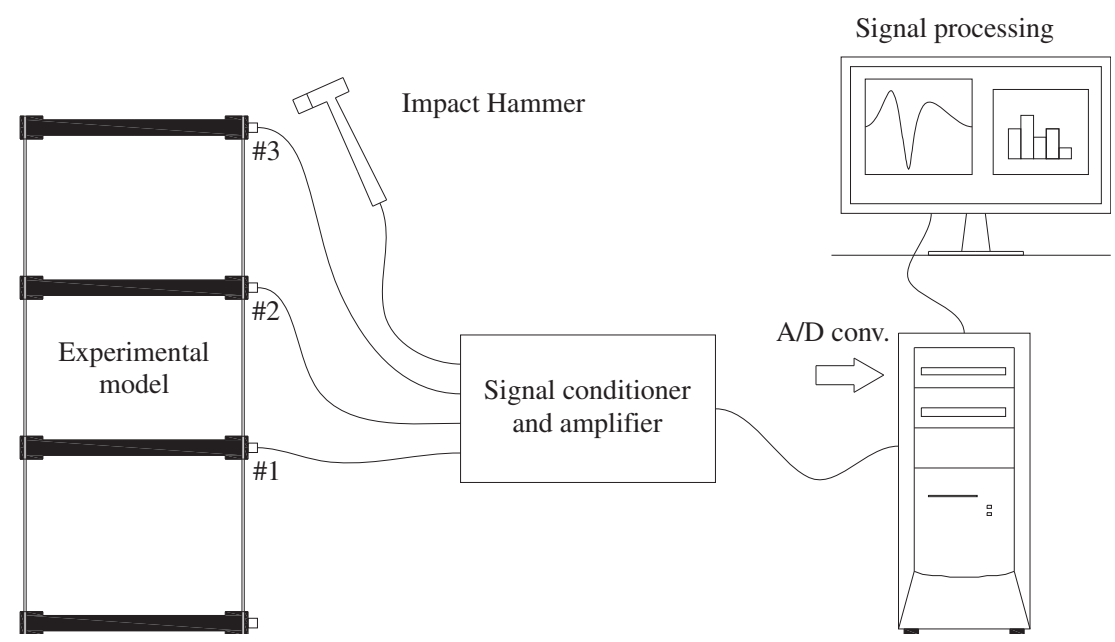

Figure 4. Acquisition system for hammer test.

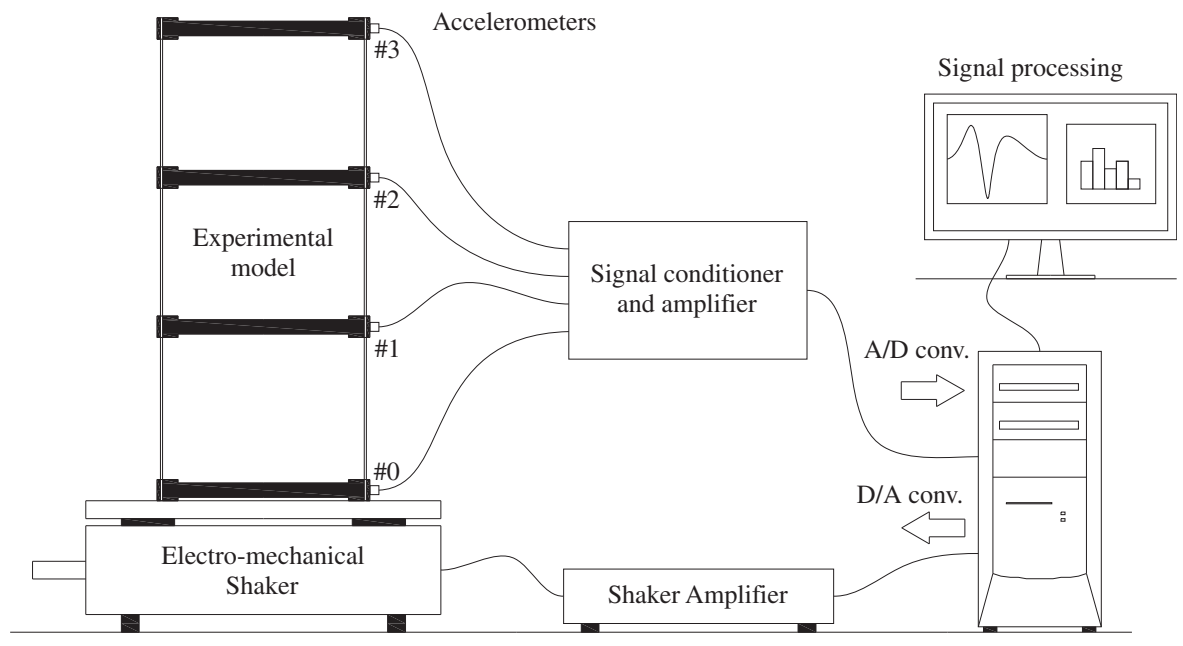

Figure 5. Acquisition system for broadband noise test. 


\section{EXPERIMENTAL RESULTS AND DISCUSSION}

To prove the robustness of the aforementioned damage identification procedure, several experimental tests have been developed, having considered different damage scenarios. For each damage scenario, both free decay tests and broadband noise tests have been performed, several samples of time history accelerations have been recorded and averaged FRF have been computed. For instance, a sample of the acquired signals for a free decay test in healthy structure is reported in Figure 6, while Figure 7 depicts the correspondent averaged FRF.

\subsection{System identification}

To identify the dynamical parameters of both healthy and damaged systems a linear system identification procedure has been used, as in the following steps:

1. Acquisition of input data (pulse force or broadband noise) and system accelerations at each floor from experimental tests;

2. Computation of the correspondent FRFs (see Figure 7);
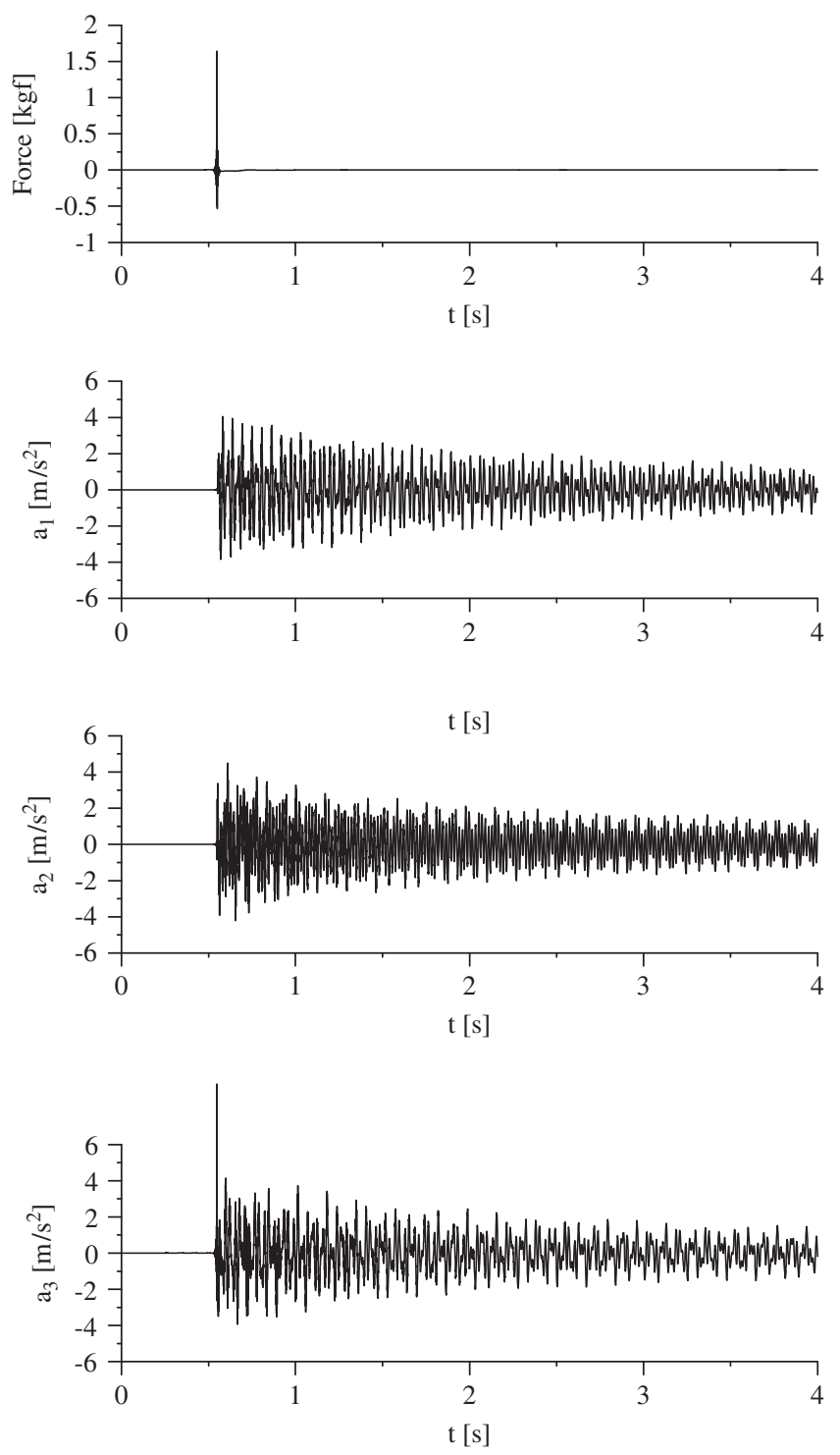

Figure 6. Signals acquired during a hammer test: impulse and floor accelerations. 


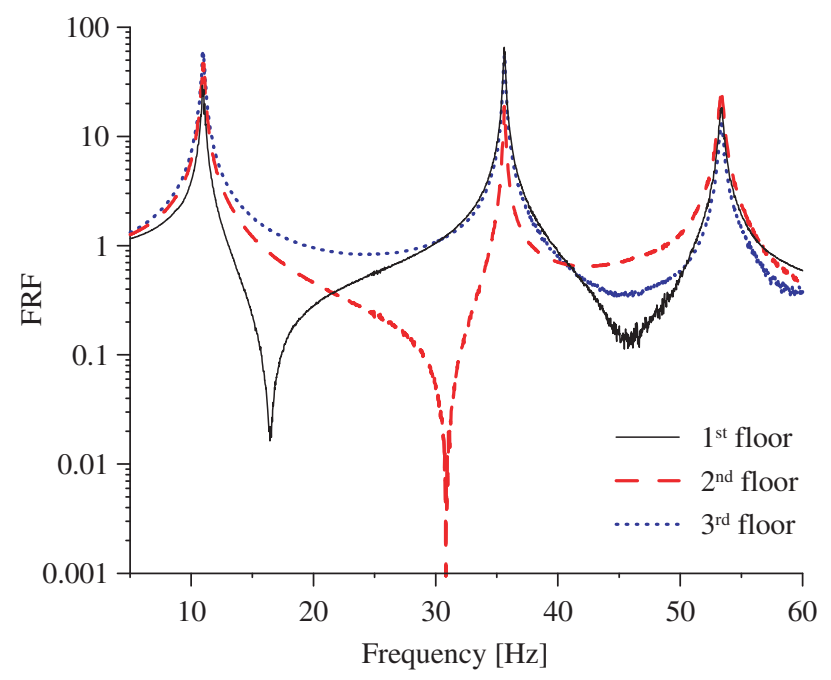

Figure 7. Signals acquired during a hammer test: FRFs.

3. Use of Rational Fraction Polynomial method (see Reference [32]) for computing natural frequencies $\omega_{i}$;

4. Solving the eigenvalues problem of the system in Equation (5), where only $M$ is known, getting the frequencies $\bar{\omega}_{i}$ depending on $k_{1}, k_{2}$ and $k_{3}\left(k_{D 1}, k_{D 2}\right.$ and $k_{D 3}$ in case of damaged system);

5. Evaluation of the objective function.

$$
J_{\bar{\omega}}\left(k_{1}, k_{2}, k_{3}\right)=\sum_{i=1}^{3} w_{i} \frac{\left(\omega_{i}-\bar{\omega}_{i}\left(k_{1}, k_{2}, k_{3}\right)\right)^{2}}{\omega_{i}^{2}}
$$

where the weighting values $w_{j}$ have been fixed to $w_{1}=1.5, w_{2}=1.0$ and $w_{3}=0.5$ in order to take into account that the first mode gives a bigger contribution to system motion than the third one;

6. Computation of the optimal values of $k_{1}, k_{2}$ and $k_{3}\left(k_{D 1}, k_{D 2}\right.$ and $k_{D 3}$ in case of damaged system) by means of an optimization algorithm based on a quasi-Newton method, and an active set strategy as well (see References [34] and [35]).

Once $k_{i}$ and $k_{D i}$ values have been determined, the expected damage values $\alpha_{i}$ may be estimated solving the equation:

$$
\alpha_{i}=\frac{k_{i}-k_{D i}}{k_{i}}, \quad i=1,3
$$

It is worth stressing that the shear-type model structure for the stiffness matrix (and consequently for the damage parameters) has been chosen in order to represent an immediate relation between numerical results and their physical meanings. Particularly, in this case, $k_{i}$ is the shear stiffness at $i$ th floor and $\alpha_{i}$ the correspondent amount of reduction resulting from damage scenarios at the same floor.

In the following the details for the damage scenarios and the results obtained by applying the above-described procedure are reported and discussed.

\subsection{Damage scenarios}

Basically the damage is realised by a reduction in the stiffness of the structure, substituting the columns of the model with others having a smaller cross section. Three damage scenarios, labelled D02, D03 and D04, have been considered and detailed in Table II. In particular, the damage scenario D02 is obtained by replacing the original columns of the first floor $(3 \times 15 \mathrm{~mm}$ 
in Figure 8(b)), with slender members, in which a reduction of area was introduced at midheight $(3 \times 7 \mathrm{~mm}$; 'dog-bone' shape in Figure $8(\mathrm{a}))$. In such a way a very low damage level is achieved only at first floor.

In the damage scenarios D03 and D04 the columns at third and second floor (Figure 8(d) in healthy structure) were respectively substituted with ones having a smaller cross section $(3 \times 10 \mathrm{~mm}$ in Figure $8(\mathrm{c}))$. These scenarios were intended to produce a larger and localized damage.

By performing the aforementioned linear system identification procedure on the healthy and damaged structure, the dynamical parameters have been obtained; in particular, from the comparison between the undamaged stiffness matrix $\mathbf{K}$ and the damaged ones $\mathbf{K}_{D}$ the expected damage values $\alpha_{i}$ are estimated. In Table III the identified stiffness at each floor and the

Table II. 'D'-type damage scenarios.

\begin{tabular}{lcccr}
\hline Cross section $(\mathrm{mm})$ & \multicolumn{3}{c}{ Damage scenarios } \\
\cline { 3 - 5 } Floor & Healthy & D02 & D03 & D04 \\
\hline $1^{\circ}$ & $3 \times 15$ & $3 \times 15^{\text {a }}$ & $3 \times 15$ & $3 \times 15$ \\
$2^{\circ}$ & $3 \times 15$ & $3 \times 15$ & $3 \times 15$ & $3 \times 10$ \\
$3^{\circ}$ & $3 \times 15$ & $3 \times 15$ & $3 \times 10$ & $3 \times 15$ \\
\hline
\end{tabular}

${ }^{\mathrm{a} D o g-b o n e ~ s h a p e d ~ c o l u m n . ~}$

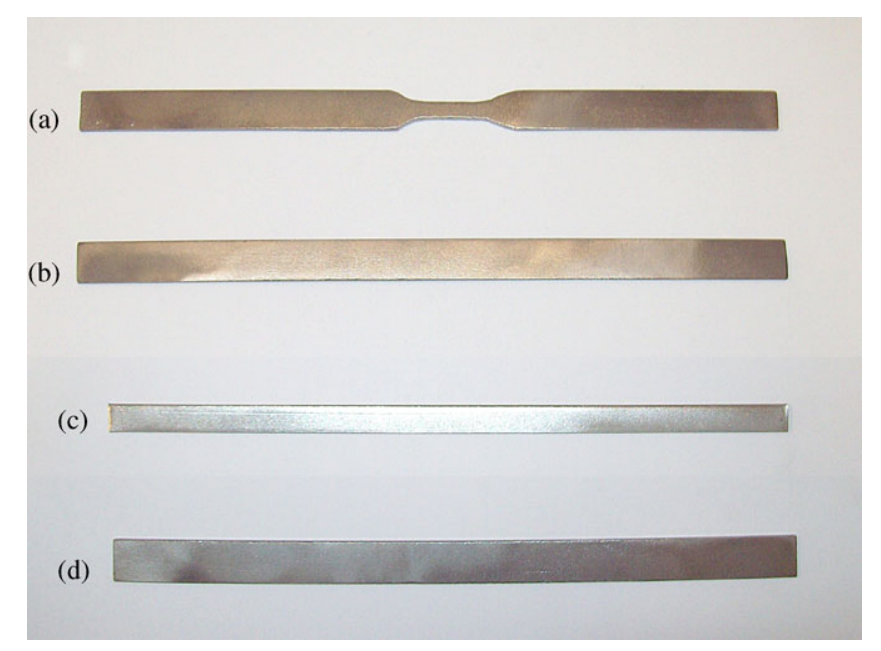

Figure 8. Columns used in different damage scenarios.

Table III. Identified stiffness and expected damage parameters for ' $\mathrm{D}$ '-type damage scenarios.

\begin{tabular}{lccrr}
\hline & \multicolumn{4}{c}{ Identified stiffness $(\mathrm{N} / \mathrm{m})$} \\
\cline { 2 - 5 } & Healthy & D02 & D03 & D04 \\
\hline$k_{1}$ & 19249.1 & 18398.4 & 19067.6 & 20484.5 \\
$k_{2}$ & 25967.1 & 26114.5 & 25305.9 & 11362.9 \\
$k_{3}$ & 26768.0 & 26744.9 & 12335.7 & 27197.1 \\
& & Expected damage parameters $(\%)$ & D04 \\
\cline { 2 - 5 } & Healthy & D02 & D03 & -6.42 \\
& - & 4.42 & 0.94 & 56.24 \\
$\alpha_{1}$ & -0.57 & 0.09 & 53.92 & -1.60 \\
$\alpha_{2}$ & - & & &
\end{tabular}


expected damage values $\alpha_{i}$ for every damage scenarios are reported.

\subsection{Hammer tests}

The systems described in Table II have been subjected to hammer tests and the recorded experimental responses have been used to evaluate the objective function as in Equations (9) and (10) in order to detect damage in terms of both localization and entity.

For hammer test the motion is governed by Equation (5) in which

$$
\mathbf{F}(t)=\mathbf{L} F_{0} \delta\left(t-t_{0}\right)
$$

where $F_{0}$ and $t_{0}$ are the impulse intensity and time instant at which impulse occurs, respectively, $\delta$ is the Dirac's delta function and $\mathbf{L}=\left[\begin{array}{lll}0 & 0 & 1\end{array}\right]^{\mathrm{T}}$ is the location vector for an impulse on the third floor. Moreover, in Equation (6) the following weighting function is assumed:

$$
w_{i}=\frac{1}{3} \quad i=1,2,3
$$

As previous works have already shown, for hammer tests the phase $\theta(t)$ and instantaneous frequency $\omega(t)=\dot{\theta}(t)$ characteristics of the analytical signal computed from the FMRs in terms of acceleration are proper to highlight damage identification. The filtering procedure has been carried out by using a cascade of eighth-order pass-band Butterworth filter, whose cut-off frequencies are accurately chosen depending on the natural frequencies.

Once the objective function has been defined, in order to find out the actual damage pattern, an optimization algorithm for the minimum search of multivariate function is adopted. This algorithm is based on a quasi-Newton method and an active set strategy to solve minimization problem. This routine computes the search direction according to the approximate definition of both local Hessian and gradient. It is also possible to specify if the variables are bounded [34].

In Figure 9 the convergence to the minimum search for objective function related to the previous case is reported. In particular in Figure 9(a) the coordinates $\alpha_{i}$ of the evolution of the minimum search algorithm starting from the initial guess as a function of iteration number are depicted, while in Figure 9(b) the correspondent value of the objective function is plotted. The objective function $J_{\theta}(\boldsymbol{\alpha})$ is computed in terms of phase of analytical signal obtained from the second floor response acceleration, case D02 for hammer test.
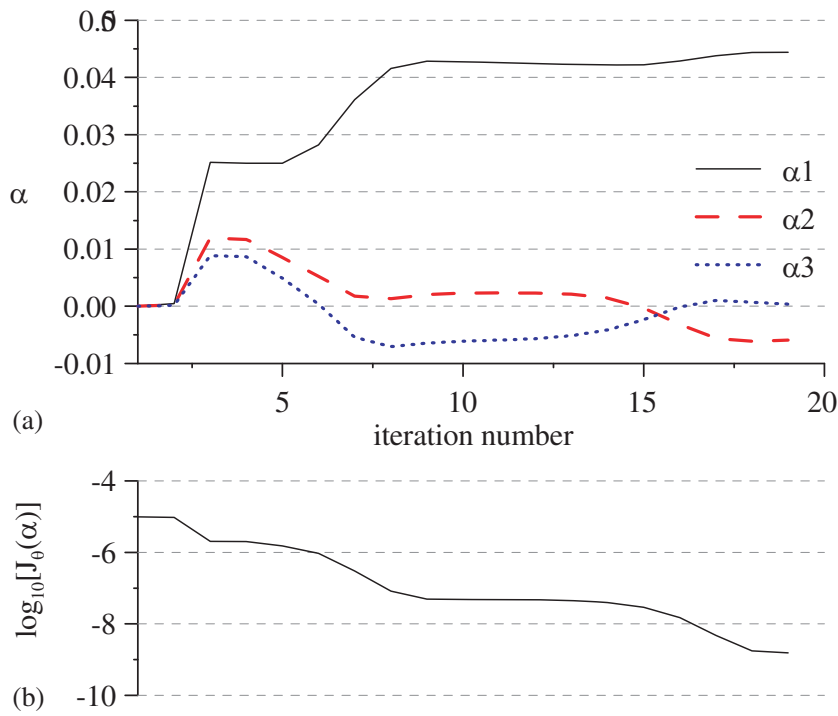

Figure 9. (a) Evolution of the coordinates $\alpha_{i}$ of the guess minimum versus the progressive iteration number and (b) correspondent value of the objective function. 
In the previous case depicted in Figure 9 the minimum can be detected at $\alpha_{1}=4.47 \%$; $\alpha_{2}=0.56 \% ; \alpha_{3}=0.02$, very close to the expected values.

The error $\varepsilon$ between the expected damage parameters and the detected ones can be synthetically expressed as a Euclidean distance in the space of the damage parameters:

$$
\epsilon=\sqrt{\sum_{i=1}^{N}\left(\alpha_{\exp , i}-\alpha_{i}\right)^{2}}
$$

where $\alpha_{\exp , i}$ are the expected damage parameters and $\alpha_{i}$ are the coordinates of the detected minimum and $N$ are the system degrees of freedom.

It has to be stressed that in this case the negative values for damage parameters are due to modal error as well as experimental errors both in structural parameters identification procedure and in damage identification procedure. It may be noted that in this case $\alpha_{2}$ and $\alpha_{3}$ are very close to zero, which is the theoretical value for the undamaged column.

For each damage scenario, a deep parametric experimental campaign was conducted in order to assess the influence of several variables upon the accuracy of the results, such as the characteristic of the analytical signal to be computed, the floor at which the response acceleration is acquired or the number of the response samples to use. These results, for clearness's sake, are summarized in the following tables. As shown in a previous work [23], it seems that in the case of free decay tests, only objective functions computed by phase or instantaneous frequency of FMRs are able to detect a sharp absolute minimum.

In Table IV the detected minima of the objective function, obtained using the phase and the instantaneous frequency characteristics, respectively, of the analytical signal are compared with the expected damage parameters. The analytical signal was computed using only one sample of the FMR of the second floor acceleration response time-history.

Although in both cases damage parameters can be accurately estimated, it is clear that for every tested damage scenario phase provides better results.

In order to investigate how the number of the repetitions of the test affects the accuracy of the results, in Table $\mathrm{V}$ the detected minima of the objective function, obtained using one and ten samples of the second floor acceleration response, are compared.

In both cases the objective function was computed by the phase characteristics of the analytical signal and it is clearly shown that the use of an averaged objective function leads to better results, even though in this case an excellent accuracy was achieved using one record only. In the authors' opinion, the more the quality of the acquired signal decreases, the more the averaging procedure becomes important to reassess the sharpness of the proposed procedure.

Table IV. Comparison between damage parameters computed from phase and instantaneous frequency characteristics extracted from a single record.

\begin{tabular}{|c|c|c|c|c|}
\hline & & Expected $(\%)$ & Phase $(\%)$ & Ist. Freq. (\%) \\
\hline \multirow[t]{4}{*}{ D02 } & $\alpha_{1}$ & 4.42 & 4.47 & 5.17 \\
\hline & $\alpha_{2}$ & -0.57 & -0.56 & -1.97 \\
\hline & $\alpha_{3}$ & 0.09 & 0.02 & 1.07 \\
\hline & & $\varepsilon(\%)$ & 0.08 & 1.87 \\
\hline \multirow[t]{4}{*}{ D03 } & $\alpha_{1}$ & 0.94 & 1.47 & 1.56 \\
\hline & $\alpha_{2}$ & 2.55 & 2.54 & 2.26 \\
\hline & $\alpha_{3}$ & 53.92 & 53.84 & 53.78 \\
\hline & & $\varepsilon(\%)$ & 0.53 & 0.69 \\
\hline \multirow[t]{4}{*}{ D04 } & $\alpha_{1}$ & -6.42 & -4.48 & -1.57 \\
\hline & $\alpha_{2}$ & 56.24 & 55.26 & 53.92 \\
\hline & $\alpha_{3}$ & -1.60 & -1.16 & 0.54 \\
\hline & & $\varepsilon(\%)$ & 2.22 & 5.78 \\
\hline
\end{tabular}


Table V. Comparison between damage parameters obtained from one and ten samples, phase characteristics, second floor.

\begin{tabular}{|c|c|c|c|c|}
\hline & & Expected $(\%)$ & 1 sample $(\%)$ & 10 samples $(\%)$ \\
\hline \multirow[t]{4}{*}{ D02 } & $\alpha_{1}$ & 4.42 & 4.47 & 4.44 \\
\hline & $\alpha_{2}$ & -0.57 & -0.56 & -0.59 \\
\hline & $\alpha_{3}$ & 0.09 & 0.02 & 0.03 \\
\hline & & $\varepsilon(\%)$ & 0.08 & 0.06 \\
\hline \multirow[t]{4}{*}{ D03 } & $\alpha_{1}$ & 0.94 & 1.47 & 1.36 \\
\hline & $\alpha_{2}$ & 2.55 & 2.54 & 2.53 \\
\hline & $\alpha_{3}$ & 53.92 & 53.84 & 53.84 \\
\hline & & $\varepsilon(\%)$ & 0.53 & 0.43 \\
\hline \multirow[t]{4}{*}{ D04 } & $\alpha_{1}$ & -6.42 & -4.48 & -4.65 \\
\hline & $\alpha_{2}$ & 56.24 & 55.26 & 55.48 \\
\hline & $\alpha_{3}$ & -1.60 & -1.16 & -1.35 \\
\hline & & $\varepsilon(\%)$ & 2.22 & 1.94 \\
\hline
\end{tabular}

Table VI. Comparison between damage parameters obtained using responses from different floors, phase characteristic, one sample.

\begin{tabular}{|c|c|c|c|c|c|}
\hline & & Expected $(\%)$ & 1st floor $(\%)$ & 2nd floor $(\%)$ & 3rd floor $(\%)$ \\
\hline \multirow[t]{4}{*}{ D02 } & $\alpha_{1}$ & 4.42 & 4.464 & 4.469 & 4.352 \\
\hline & $\alpha_{2}$ & -0.57 & -0.575 & -0.560 & -0.319 \\
\hline & $\alpha_{3}$ & 0.09 & 0.038 & 0.020 & -0.031 \\
\hline & & $\varepsilon(\%)$ & 0.066 & 0.083 & 0.283 \\
\hline \multirow[t]{4}{*}{ D03 } & $\alpha_{1}$ & 0.94 & 1.468 & 1.471 & 3.145 \\
\hline & $\alpha_{2}$ & 2.55 & 2.531 & 2.538 & -3.002 \\
\hline & $\alpha_{3}$ & 53.92 & 53.839 & 53.840 & 52.170 \\
\hline & & $\varepsilon(\%)$ & 0.532 & 0.534 & 6.220 \\
\hline \multirow[t]{4}{*}{ D04 } & $\alpha_{1}$ & -6.42 & -8.418 & -4.482 & -5.662 \\
\hline & $\alpha_{2}$ & 56.24 & 52.549 & 55.258 & 55.845 \\
\hline & $\alpha_{3}$ & -1.60 & -2.624 & -1.161 & -2.051 \\
\hline & & $\varepsilon(\%)$ & 4.320 & 2.216 & 0.964 \\
\hline
\end{tabular}

Furthermore, the considered procedure has the desirable feature that damage location and quantification can be determined for any of the measured points, as clearly shown in Table VI in which the detected minima of the objective function, obtained using one sample of the acceleration response at each floor, are reported.

\subsection{Broadband noise tests}

In order to prove the effectiveness of the proposed procedure even in case of randomly generated excitations, the systems described in Table II have also been subjected to broadband noise tests and the recorded experimental responses have been used to evaluate the objective function as in Equations (9) and (10), in order to detect damage in terms of both localization and entity. In all tests the broadband noises are generated in such a way that the power spectral density functions are approximately constant in the range from 5 to $80 \mathrm{~Hz}$.

For broadband noise tests the motion is governed by Equation (5) in which

$$
\mathbf{F}(t)=-\mathbf{M L} \ddot{x}_{0}(t)
$$


where $\ddot{x}_{0}(t)$ is the recorded ground acceleration and $\mathbf{L}=\left[\begin{array}{lll}1 & 1 & 1\end{array}\right]^{\mathrm{T}}$ is the location vector. The same weighting function reported in (14) is assumed.

As previous works have already shown [9-12], when the system is forced by a ground acceleration both FMRs and the amplitude $A(t)$ characteristic of the analytical signal computed from the FMRs in terms of acceleration are able to perform damage identification. Moreover, the averaging of objective function computed from several samples of the response is required.

Even for broadband noise, for each damage scenario, a deep parametric experimental campaign was conducted in order to assess the influence of several variables upon the accuracy of the results, such as the characteristic of the analytical signal to be computed, or the floor at which the response acceleration is acquired. These results, for clearness's sake, are summarized in the following tables.

In Table VII the minima of the objective function, obtained using the filtered modal components and the amplitude of their analytical signal, are compared with the expected damage parameters. The analytical signal was computed using six consecutive records of the second floor acceleration response time-history. Even in these cases, damage parameters can accurately be estimated both using amplitude and filtered modal components. Moreover, the same remarks about accuracy for ' $D$ '-type scenarios still holds true. The positive feature that

Table VII. Comparison between damage parameters computed from filtered modal components and amplitude characteristic; second floor, six consecutive records.

\begin{tabular}{|c|c|c|c|c|}
\hline & & Expected $(\%)$ & FMR $(\%)$ & Amplitude (\%) \\
\hline \multirow{4}{*}{$\overline{\mathrm{D} 02}$} & $\alpha_{1}$ & 4.42 & 4.70 & 4.66 \\
\hline & $\alpha_{2}$ & -0.57 & -1.94 & -2.14 \\
\hline & $\alpha_{3}$ & 0.09 & 1.28 & 1.38 \\
\hline & & $\varepsilon(\%)$ & 1.84 & 2.05 \\
\hline \multirow{4}{*}{ D03 } & $\alpha_{1}$ & 0.94 & 1.01 & 0.98 \\
\hline & $\alpha_{2}$ & 2.55 & 2.43 & 2.38 \\
\hline & $\alpha_{3}$ & 53.92 & 53.97 & 53.93 \\
\hline & & $\varepsilon(\%)$ & 0.15 & 0.18 \\
\hline \multirow[t]{4}{*}{ D04 } & $\alpha_{1}$ & -6.42 & -5.68 & -5.65 \\
\hline & $\alpha_{2}$ & 56.24 & 58.49 & 57.68 \\
\hline & $\alpha_{3}$ & -1.60 & -1.95 & -1.47 \\
\hline & & $\varepsilon(\%)$ & 2.39 & 1.64 \\
\hline
\end{tabular}

Table VIII. Comparison between damage parameters obtained using responses from different floors, amplitude characteristic, six consecutive records.

\begin{tabular}{rrrrrr}
\hline & & Expected (\%) & 1st floor (\%) & 2nd floor (\%) & 3rd floor (\%) \\
\hline D02 & $\alpha_{1}$ & 4.42 & 4.594 & 4.662 & 4.707 \\
& $\alpha_{2}$ & -0.57 & -1.809 & -2.137 & -1.592 \\
& $\alpha_{3}$ & 0.09 & 1.148 & 1.384 & 0.956 \\
\cline { 3 - 6 } & & $\varepsilon(\%)$ & 1.638 & 2.047 & 1.369 \\
\cline { 3 - 6 } D03 & $\alpha_{1}$ & 0.94 & 1.062 & 0.982 & 0.529 \\
& $\alpha_{2}$ & 2.55 & 2.368 & 2.379 & 2.462 \\
& $\alpha_{3}$ & 53.92 & 53.927 & 53.931 & 53.997 \\
\cline { 3 - 6 } D04 & & $\varepsilon(\%)$ & 0.220 & 0.177 & 0.427 \\
& & -6.42 & -6.541 & -5.650 & -6.871 \\
& $\alpha_{1}$ & 56.24 & 58.068 & 57.680 & 56.365 \\
& $\alpha_{2}$ & -1.60 & -1.974 & -1.470 & -0.884 \\
\hline & $\alpha_{3}$ & $\varepsilon(\%)$ & 1.870 & 1.638 & 0.856 \\
\hline
\end{tabular}


damage patterns can be identified from any measurement points, as described in the previous section, is valid also for broadband noise tests. This is clearly shown in Table VIII in which the detected minima of the objective function, obtained using six samples of the acceleration response at each floor, are compared. The accuracy of the result seems not to be affected by the choice of the floor.

\section{CONCLUSIONS}

In this paper the damage identification using data records in dynamic setting has been investigated. Previous works defined a damage identification procedure based on the use of the Hilbert Transform and the so-called analytical signal obtained from the responses of a sheartype model to both pulse force and environmental noise loads. Amplitude and phase of analytical signal proved to be very sensitive to small variation of system parameters and allow the detection and estimation of damage, even at early stage.

In this work the damage identification procedure is validated by means of experimental tests. A three-degree-of-freedom shear-type model, built up in the laboratory of the Structural, Aerospace and Geotechnical Engineering Department of University of Palermo, subjected to several damage scenarios has been analyzed and the obtained results show the effectiveness of the method in accordance with those obtained numerically.

In particular, the proposed procedure has the desirable feature that damage location and entity can be determined for any of the measured points. In most cases the accuracy of the results is only slightly affected by the choice of the measurement point. Furthermore, a very small number of response samples are required to correctly detect damage patterns.

\section{REFERENCES}

1. Adams RD, Cawley, Pye CJ, Stone BJ. A vibration technique for non-destructively assessing the integrity of structures. Journal of Mechanical Engineering Science 1991; 20:93-100.

2. Pabst U, Hagedorn P. On the identification of localized losses of stiffness in structures, American Society of Mechanical Engineers, DE 1993; 59:99-104.

3. Cerri MN, Vestroni F. Detection of damage in beams subjected to diffused cracking. Journal of Sound and Vibration 2000; 234:259-276.

4. Lee BT, Sun TC, Liu D. An assessment of damping measurements in the evaluation of integrity of composite beams. Journal of Reinforced Plastics and Composites 1987; 6:114-125.

5. Davini C, Gatti F, Morassi A. A damage analysis of steel beams. Meccanica 1992; 22:321-332.

6. Gounaris GD, Papadopoulus CA, Dimarogonas AD. Crack identification in beams by coupled response measurements. Computers and Structures 1996; 58:299-302.

7. Pandey AK, Biswas M, Samman MM. Damage detection from changes in curvature mode shapes. Journal of Sound and Vibration 1991; 145:321-332.

8. Luo H, Hanagud S. An integral equation for changes in the structural dynamics characteristics of damaged structures. International Journal of Solids and Structures 1997; 34:4557-4579.

9. Ratcliffe CP. A frequency and curvature based experimental method for locating damage in structure. Journal of Vibration Acoustics 1999; 226:1029-1042.

10. Wang Z, Lin RM, Lim MK. Structural damage detection using measured FRF data. Computer Methods in Applied Mechanics 1997; 147:187-197.

11. Thyagarajan SK, Schulz MJ, Pai PF. Detecting structural damage using frequency response functions. Journal of Sound and Vibration 1998; 210:162-170.

12. Sampaio RPC, Maya NMM, Silva JMM. Damage detection using frequency-response-function curvature method. Journal of Sound and Vibration 1999; 226:1029-1042.

13. Lee U, Shin J. A frequency response function-based structural damage identification method. Computers and Structures 2002; 80:117-132.

14. Shi ZY, Law SS, Zhang LM. Structural damage localization from modal strain energy changes. Journal of Sound and Vibration 1998; 218:825-844.

15. Cornwell P, Doebling SW, Farrar CR. Application of the strain energy damage detection method to plate-like structures. Journal of Sound and Vibration 1999; 224:359-374.

16. Rodden WP. A method for deriving structural influence coefficients from ground vibration test. AIAA Journal 1967; 5:991-1000.

17. Kabe AM. Stiffness matrix adjustment using mode data. AIAA Journal 1985; 28:1431-1436.

18. Abdalla MO, Grigoriadis KM, Zimmerman DC. Structural damage detection using linear matrix inequality methods. Journal of Vibration and Acoustics 2000; 122:448-454. 
19. Collins JD, Hart GC, Hasselman TK, Kennedy B. Statistical identification of structures. AIAA Journal 1974; 12:185-190.

20. Manning RA. Structural damage detection using active members and neural networks. AIAA Journal 1994; 32:1331-1333.

21. Rhim J, Lee SW. A neural network approach for damage detection and identification of structures. Computational Mechanics 1995; 16:437-443.

22. Cottone G, Pirrotta A, Salamone S. Incipient damage identification through characteristics of the analytical signal response. Structural Control and Health Monitoring 2008; 15:1122-1142.

23. Barone G, Marino F, Pirrotta A. Low stiffness variation in structural systems: identification and localization. Structural Control and Health Monitoring 2008; 15:450-470.

24. Barone G, Di Paola M, Pirrotta A. A further insight on the intrinsic mode function through stochastic analysis. Proceedings of Eurodyn, Paris, France, 4-7 September 2005.

25. Di Paola M, Navarra G, Pirrotta A. Damage detection based on the analytical signal representation. 4 WCSCM 4th World Conference on Structural Control and Monitoring, San Diego, CA, U.S.A., 2006.

26. Huang NE, Shen Z, Long SR, Wu MLC, Shih EH, Zheng Q, Tung CC, Liu HH. The empirical mode decomposition and the Hilbert-Huang spectrum for nonlinear and non-stationary time series analysis. Proceedings of the Royal Society of London A 1998; 454:903-995.

27. Montesinos ME, Munoz-Cobo JL, Perez C. Hilbert-Huang analysis of BWR neutron detector signals: application to DR calculation and to corrupted signal analysis. Annals of Nuclear Energy 2003; 30:715-727.

28. Xu YL, Chen J. Structural damage using empirical mode decomposition: experimental investigation. Journal of Engineering Mechanics (ASCE) 2004; 130:1279-1288.

29. Yang JN, Lei Y, Lin S, Huang NE. Hilbert-Huang based approach for structural damage detection. Journal of Engineering Mechanics (ASCE) 2004; 130:85-95.

30. Yang JN, Lei Y. System identification of linear structures using Hilbert transform end empirical mode decomposition. Proceedings of the $18^{\text {th }}$ International Modal Analysis Conference: A Conference on Structural Dynamics, San Antonio, TX, vol. 1, 2000; 213-219.

31. Yang JN, Lei Y, Pan S, Huang NE. System identification of linear structures based on Hilbert-Huang spectral analysis. Part 1: normal modes. Earthquake Engineering and Structural Dynamics 2003; 32:1443-1467.

32. Richardson MH, Formenti DL. Parameter estimation from frequency response measurement using rational fraction polynomials, 1st IMAC Conference, Orlando, FL, U.S.A., 1982.

33. Ibrahim SR, Mikulcik EC. A method for the direct identification of vibration parameters from the free response. The Shock and Vibration Bulletin 1977; 47(4):183-198.

34. Dennis Jr JE, Schnabel RB. Numerical Methods for Unconstrained Optimization and Nonlinear Equations. Prentice-Hall: Englewood Cliffs, NJ, 1983.

35. Gill PE, Murray W. Minimization subject to bounds on the variables. NPL Report NAC 72, National Physical Laboratory, England, 1976. 